\title{
On a System of Reaction-Diffusion Equations Arising From Competition in an Unstirred Chemostat
}

\author{
Sze-Bi Hsu; P. Waltman \\ SIAM Journal on Applied Mathematics, Vol. 53, No. 4. (Aug., 1993), pp. 1026-1044.
}

Stable URL:

http://links.jstor.org/sici?sici=0036-1399\%28199308\%2953\%3A4\%3C1026\%3AOASORE\%3E2.0.CO\%3B2-M

SIAM Journal on Applied Mathematics is currently published by Society for Industrial and Applied Mathematics.

Your use of the JSTOR archive indicates your acceptance of JSTOR's Terms and Conditions of Use, available at http://www.jstor.org/about/terms.html. JSTOR's Terms and Conditions of Use provides, in part, that unless you have obtained prior permission, you may not download an entire issue of a journal or multiple copies of articles, and you may use content in the JSTOR archive only for your personal, non-commercial use.

Please contact the publisher regarding any further use of this work. Publisher contact information may be obtained at http://www.jstor.org/journals/siam.html.

Each copy of any part of a JSTOR transmission must contain the same copyright notice that appears on the screen or printed page of such transmission.

The JSTOR Archive is a trusted digital repository providing for long-term preservation and access to leading academic journals and scholarly literature from around the world. The Archive is supported by libraries, scholarly societies, publishers, and foundations. It is an initiative of JSTOR, a not-for-profit organization with a mission to help the scholarly community take advantage of advances in technology. For more information regarding JSTOR, please contact support@jstor.org. 


\title{
ON A SYSTEM OF REACTION-DIFFUSION EQUATIONS ARISING FROM COMPETITION IN AN UNSTIRRED CHEMOSTAT*
}

\author{
SZE-BI HSU† AND P. WALTMAN $\ddagger$
}

\begin{abstract}
A system of reaction-diffusion equations modeling two-species competition in an unstirred chemostat is considered. The asymptotic behavior of solutions is given as a function of the parameters, and it is determined when neither, one, or both competing populations survive. Techniques include the maximum principle, theory of uniform persistence in infinite-dimensional dynamical systems, and the theory of strongly order-preserving semidynamical system.
\end{abstract}

Key words. chemostat, maximum principle, uniform persistence, infinite-dimensional system, strongly order-preserving semiflow

AMS(MOS) subject classification. 92A 17

1. Introduction. The chemostat has a well-established role as a model open system in ecology as attested to in the survey articles [FS], [HET], [JM], [V], [W2], and [WHH]. In the simplest case, the theory [HHW], [Hsu ], [W1], and experiments [HH] are in agreement. Basically, the chemostat consists of a nutrient input-with all nutrients needed for growth in abundance except one-pumped at a constant rate into a wellmixed culture vessel whose volume is kept constant by pumping the contents out at the same rate. In addition to being a piece of laboratory apparatus for the continuous culturing of bacteria, it is a model for a very simple lake where exploitative competition is easily studied. The input concentration of nutrient $S^{(0)}$ and the washout rate $D$ are assumed constant and are under the control of the experimenter. If $S(t)$ is the nutrient concentration at time $t$, and if $u(t), v(t)$ are the concentration of the competing populations, the model is given by [HET], [TW], [WHH]

$$
\begin{gathered}
\frac{d S}{d t}=\left(S^{(0)}-S\right) D-\frac{m_{1} S u}{a_{1}+S}-\frac{m_{2} S v}{a_{2}+S}, \\
\frac{d u}{d t}=\left(\frac{m_{1} S}{a_{1}+S}-D\right) u, \\
\frac{d v}{d t}=\left(\frac{m_{2} S}{a_{2}+S}-D\right) v, \\
S(0) \geqq 0, \quad u(0) \geqq 0, \quad v(0) \geqq 0,
\end{gathered}
$$

where $m_{i}$ is the maximal growth rate and $a_{i}$ is the Michaelis-Menten (or half-saturation) constant. The mathematical analysis shows that only one of the populations $u$ or $v$ survives. However, the coexistence of competing populations is obvious in nature. A candidate for an explanation is to remove the "well-mixed" hypothesis.

A piece of laboratory apparatus designed for this purpose by Lovitt and Wimpenny [LW] is called a gradostat. The mathematical analysis of this apparatus has been the subject of several papers [T], [JSTW], [STW], and a survey of the general results is given in [SW]. The simple gradostat consists of $n$ vessels with constant nutrient input

* Received by the editors December 23, 1991; accepted for publication (in revised form) July 1, 1992.

$\dagger$ Institute of Applied Mathematics, National Tsing-Hua University, Hsinchu, Taiwan. This author's research was supported by National Council of Science, Republic of China.

$\ddagger$ Department of Mathematics and Computer Science, Emory University, Atlanta, Georgia 30345. This author's research was supported by National Science Foundation grant 89-01992. 
into the left vessel and output in the leftmost and rightmost vessels. The contents of the vessel are pumped into the nearest neighboring vessels at the same constant rate. This creates a "discrete" nutrient gradient. The equations take the following form:

$$
\begin{gathered}
\frac{d S_{i}}{d t}=\left(S_{i-1}-2 S_{i}+S_{i+1}\right) D-u_{i} f_{u}\left(S_{i}\right)-v_{i} f_{v}\left(S_{i}\right), \\
\frac{d u_{i}}{d t}=\left(u_{i-1}-2 u_{i}+u_{i+1}\right) D+u_{i} f_{u}\left(S_{i}\right), \\
\frac{d v_{i}}{d t}=\left(v_{i-1}-2 v_{i}+v_{i+1}\right) D+v_{i} f_{v}\left(S_{i}\right),
\end{gathered}
$$

$$
i=1,2, \ldots, n \text {, }
$$

where

$$
\begin{gathered}
f_{u}(S)=\frac{m_{u} S}{a_{u}+S}, \quad f_{v}(S)=\frac{m_{v} S}{a_{v}+S}, \\
S_{0}=S^{(0)}, \quad u_{0}=v_{0}=0, \\
S_{n+1}=u_{n+1}=v_{n+1}=0 ;
\end{gathered}
$$

$S_{i}(t), u_{i}(t), v_{i}(t)$ are the concentration of nutrient, competing species at the $i$ th vessel.

Another alternative is simply to use one vessel and remove the "well-stirred" hypothesis of the basic chemostat yielding a system of reaction-diffusion equations of the following form (assuming equal diffusion rates, see $\S 5$ for comments):

$$
\begin{aligned}
& \frac{\partial S}{\partial t}=d \frac{\partial^{2} S}{\partial x^{2}}-\frac{m_{1} S u}{a_{1}+S}-\frac{m_{2} S v}{a_{2}+S}, \\
& \frac{\partial u}{\partial t}=d \frac{\partial^{2} u}{\partial x^{2}}+\frac{m_{1} S u}{a_{1}+S}, \\
& \frac{\partial v}{\partial t}=d \frac{\partial^{2} v}{\partial x^{2}}+\frac{m_{2} S v}{a_{2}+S}, \quad 0<x<1,
\end{aligned}
$$

with boundary conditions

$$
\begin{aligned}
& \frac{\partial S}{\partial x}(t, 0)=-S^{(0)}, \\
& \frac{\partial u}{\partial x}(t, 0)=\frac{\partial v}{\partial x}(t, 0)=0,
\end{aligned}
$$

$$
\begin{aligned}
& \frac{\partial S}{\partial x}(t, 1)+r S(t, 1)=0, \\
& \frac{\partial u}{\partial x}(t, 1)+r u(t, 1)=0, \\
& \frac{\partial v}{\partial x}(t, 1)+r v(t, 1)=0
\end{aligned}
$$


and initial conditions

$$
\begin{array}{ll}
S(0, x)=S_{0}(x) \geqq 0, & \\
u(0, x)=u_{0}(x) \geqq 0, & u_{0}(x) \not 0, \\
v(0, x)=v_{0}(x) \geqq 0, & v_{0}(x) \not 0 .
\end{array}
$$

The steady-state behavior of this system was investigated in [SoW]. Standard bifurcation theorems were used to show coexistence, but without any stability results. In this paper, we investigate the above reaction-diffusion system as a dynamical system, and we do obtain some stability results. The point of view is much like that of [DRS], particularly as reinterpreted in [HS, § 5], where strong use of the persistence theorem of [ HW] is used. The paper may be considered a dynamical extension of the steady-state results in [SoW]. We note that [SoW] shows that the "steady states" are not constant functions, but space-dependent, as we would anticipate from the boundary conditions.

The boundary conditions in (1.2) are fairly intuitive and appropriate for this type of equation; a derivation of the condition at the left-hand end was given in [SoW], and the condition at the other end follows similarly. However, the derivative conditions are not clearly defined in terms of the operating parameters and are not directly related to the parameters of the original basic chemostat model. It is instructive to consider the problem in a heuristic way to see how the units compare between the basic chemostat and the chemostat without the assumption of well mixing. To keep matters simple, we consider only the nutrient equation without consumption (equivalently, zero initial conditions for the microorganisms). The basic chemostat takes the form $S^{\prime}(t)=\left(S^{(0)}-\right.$ $S(t)) D$. The units of $S$ are concentration, mass/volume. The total mass of substrate is $V S$, where $V$ is the volume of the vessel; if $F$ is the flow rate (the rate of the pump operating the chemostat), the parameter $D$ is defined as $F / V$. Rewriting the above equation for the mass of the substrate in the vessel yields

$$
V S^{\prime}(t)=F S^{(0)}-F S(t) .
$$

Equation (1.4) simply says that the rate of change in mass is proportional to the difference between the incoming flux and the outgoing flux.

When switching to the partial differential equation (PDE), the basic quantity $S(t, x)$ becomes a density measured in units of mass per unit length. The nutrient equation for the unstirred chemostat is governed by

$$
S_{t}=d S_{x x}
$$

Integrate over the interval $[0,1]$ to obtain an equation for the total mass of nutrient,

$$
\frac{d}{d t} \int_{0}^{1} S(t, x) d x=d S_{x}(t, 1)-d S_{x}(t, 0) .
$$

The two terms on the right-hand side represent the flux at the right and left endpoints, respectively, and so (1.6) is the counterpart of (1.4). These quantities should be given by the boundary conditions. The flux at the left end is given by $\bar{S}^{(0)} F$, where $\bar{S}^{(0)}$ corresponds to the $S^{(0)}$ of the basic chemostat (as a density, i.e., the units are $\mathrm{m} / \mathrm{l}$ ). The condition at the left endpoint can be written as

$$
d S_{x}(t, 0)=-\bar{S}^{(0)} F
$$

or if we define (a slight abuse of terminology)

$$
S^{(0)}=\frac{\bar{S}^{(0)} F}{d}
$$


the first boundary condition in (1.2) is obtained. Similarly, the flux at the right-hand end is given by $d S_{x}(t, 1)=F S(t, 1)$. Thus, if $r$ is defined by $r=F / d$, then the second boundary condition holds.

Equation (1.6) shows that the rate of change of the mass of the nutrient in the vessel is proportional to the difference between the input nutrient flux and the output nutrient flux, just like the basic chemostat. The diffusion coefficient $d$ has units length squared over time, $l^{2} / t$. Thus the units match.

2. Simplification. The following basic lemma allows the problem to be simplified.

LEMMA 2.1. The solutions $S(t, x), u(t, x), v(t, x)$ of $(1.1)-(1.3)$ exist for all $t>0,0<x<1$, are nonnegative, bounded, and

$$
\|S(t, \cdot)+u(t, \cdot)+v(t, \cdot)-\phi\|_{\infty}=\mathcal{O}\left(e^{-\alpha t}\right) \text { as } t \rightarrow \infty
$$

for some $\alpha>0$, where

$$
\phi=\phi(x)=S^{(0)}\left(\frac{1+r}{r}-x\right), \quad 0<x<1 .
$$

Proof. Local existence is standard [Sm, p. 193]. The positivity of solutions can be established by showing that the region $\{(S, u, v) \mid S \geqq 0, u \geqq 0, \nu \geqq 0\}$ is invariant [CCS] and by using the strong maximum principle [ $\mathrm{L}$, p. 53].

Let $w(t, x)=S(t, x)+u(t, x)+v(t, x)-\phi(x)$. Adding the equations, initial conditions and boundary conditions yield that $w(t, x)$ satisfies

$$
\begin{gathered}
\frac{\partial w}{\partial t}=d \frac{\partial^{2} w}{\partial x^{2}}, \\
\frac{\partial w}{\partial x}(t, 0)=0, \\
\frac{\partial w}{\partial x}(t, 1)+r w(t, 1)=0, \\
w(0, x)=S(0, x)+w(0, x)+v(0, x)-\phi(x) .
\end{gathered}
$$

Let $\eta_{0}>0$ and $\psi=\psi(x)>0$ be the least eigenvalue and principal eigenfunction, respectively, for

$$
\begin{gathered}
d \psi^{\prime \prime}+\lambda \psi=0, \\
\psi^{\prime}(0)=0, \quad \psi^{\prime}(1)+r \psi(1)=0 .
\end{gathered}
$$

It follows from [CL, p. 212] that $\psi(x)>0$ on $(0,1)$. However, uniqueness of solutions of initial value problems allow us to conclude that $\psi(0)>0$ and $\psi(1)>0$ or $\psi(x)>0$ on $[0,1]$ as claimed.

We seek to solve (2.3)-(2.6) by separation of variables. Following [L], let

$$
w(x, t)=\psi(x) z(x, t) e^{-\alpha t},
$$

where $\alpha>0$ is to be determined. It follows easily that $z(x, t)$ satisfies

$$
\begin{gathered}
d \frac{\partial^{2} z}{\partial x^{2}}-\frac{\partial z}{\partial t}+2 d \frac{\psi^{\prime}}{\psi} \frac{\partial z}{\partial x}+\left(-\eta_{0}+\alpha\right) z=0, \\
\frac{\partial z}{\partial x}(t, 0)=0, \quad \frac{\partial z}{\partial x}(t, 1)=0 .
\end{gathered}
$$


Let $\alpha$ satisfy $0<\alpha<\eta_{0}$. Then using (2.10), (2.11), and the maximum principle, it follows that $z(t, x)$ has no nonnegative maximum $M$ at interior point unless $M=0$. Hence

$$
z(t, x) \leqq \max _{0 \leqq x \leqq 1} z(0, x) .
$$

Similarly, replacing $z$ by $-z$ in (2.10) and (2.11) yields that

$$
-z(t, x) \leqq \max _{0 \leqq x \leqq 1}(-z(0, x)) \quad \text { or } \quad z(t, x) \geqq-\min _{0 \leqq x \leqq 1} z(0, x) .
$$

Hence, (2.10) and (2.11) imply that $|z(t, x)| \leqq C$ for some $C>0$. Equation (2.1) now follows from (2.9), and the lemma is established.

The function $\phi(x)$ represents the distribution of nutrient in the case where there was no consumption $\left(u_{0}(x) \equiv 0, v_{0}(x) \equiv 0\right)$. The lemma reflects the fact that the total nutrient and equivalent organism biomass equilibrate to this function as well. This is essentially a definition of the chemostat if all variables are taken into account. The parameters $S^{(0)}$ and $r$ are reflected in the function $\phi(x)$. These are the operating parameters of the chemostat.

Solutions of (1.1)-(1.3) generate a semidynamical system on $C_{+} \times C_{+} \times C_{+}$, where $C_{+}$is the set of nonnegative, continuous functions on $[0,1]$ with the usual supremum norm. This semidynamical system is denoted by $T(t) x$, where $t \geqq 0$ and $x$ represents the triple of initial conditions given by (1.3). For $t>0$, the operator is compact [H]. The lemma shows that the system is dissipative and hence has a connected global attractor [H, p. 19]. Equation (2.1) allows us to conclude that the attractor (and hence all omega limit sets) lies in the subset given by $S+u+v-\phi=0$. On this set, (1.1)-(1.3) becomes

$$
\begin{gathered}
\frac{\partial u}{\partial t}=d \frac{\partial^{2} u}{\partial x^{2}}+f_{1}(\phi-u-v) u, \\
\frac{\partial v}{\partial t}=d \frac{\partial^{2} v}{\partial x^{2}}+f_{2}(\phi-u-v) v \\
\frac{\partial u}{\partial x}(t, 0)=0, \quad \frac{\partial u}{\partial x}(t, 1)+r u(t, 1)=0, \\
\frac{\partial v}{\partial x}(t, 0)=0, \quad \frac{\partial v}{\partial x}(t, 1)+r v(t, 1)=0 \\
u(0, x)=u_{0}(x) \geqq 0, \quad v(0, x)=v_{0}(x) \geqq 0, \\
u_{0}(x) \neq 0, \quad v_{0}(x) \neq 0, \quad \phi(x)-u_{0}(x)-v_{0}(x) \geqq 0
\end{gathered}
$$

where

$$
f_{i}(S)=\left[\begin{array}{ll}
m_{i} S /\left(a_{i}+S\right), & S \geqq 0, \\
0, & S \leqq 0,
\end{array} \quad i=1,2 .\right.
$$

This is the system that is investigated in the following sections. Theorem 4.1 of [ Th] connects the dynamics of (1.1)-(1.3) to the dynamics of system (2.13)-(2.15) whenever we are able to show the existence of a stable attractor for (2.13)-(2.15).

3. Single population growth and extinction results. As noted in the Introduction, the parameters $S^{(0)}$ and $r$ are under the control of the experimenter. The constants $m_{i}$ and $a_{i}, i=1,2$, represent properties of the organisms. Equal diffusion is a simplification. 
It is important to know that the two classes of organisms are viable under the chemostat's operating parameters without competition. The population of each must be able to survive alone in the chemostat if it is to be able to survive with a competitor.

If the initial condition $v_{0}(x) \equiv 0$, then a lower-dimensional dynamical system results, formally equivalent to setting $v(t, x) \equiv 0$ in (1.1). Since Lemma 2.1 still holds, studying the growth of a single population is equivalent to setting $v(t, x) \equiv 0$ in (2.13). Thus it is appropriate to consider

$$
\begin{gathered}
\frac{\partial u}{\partial t}=d \frac{\partial^{2} u}{\partial x^{2}}+\frac{m_{1}(\phi(x)-u)}{a_{1}+\phi(x)-u} u \\
\frac{\partial u}{\partial x}(t, 0)=0, \quad \frac{\partial u}{\partial x}(t, 1)+r u(t, 1)=0 .
\end{gathered}
$$

If $u(t, x) \equiv 0$ in $(2.13)$, then we have

$$
\begin{gathered}
\frac{\partial v}{\partial t}=d \frac{\partial^{2} v}{\partial x^{2}}+\frac{m_{2}(\phi(x)-v)}{a_{2}+\phi(x)-v} v, \\
\frac{\partial v}{\partial x}(t, 0)=0, \quad \frac{\partial v}{\partial x}(t, 1)+r v(t, 1)=0 .
\end{gathered}
$$

The following theorem provides conditions under which an organism cannot survive in the given environment, that is, given the fixed washout rate $r$ and the fixed input concentration $S^{(0)}$.

THEOREM 3.1. (i) If $m_{1}<\lambda_{0} d$, then $u(x, t)$ decays to zero exponentially as $t \rightarrow$ $\infty$, where $\lambda_{0}>0$ is the first eigenvalue of

$$
\begin{gathered}
\psi^{\prime \prime}+\lambda\left(\frac{\phi(x)}{a_{1}+\phi(x)}\right) \psi=0, \\
\psi^{\prime}(0)=0, \quad \psi^{\prime}(1)+r \psi(1)=0 .
\end{gathered}
$$

(ii) If $m_{2}<\mu_{0} d$, then $v(x, t)$ decays to zero exponentially as $t \rightarrow \infty$, where $\mu_{0}>$ 0 is the first eigenvalue of

$$
\begin{gathered}
\psi^{\prime \prime}+\mu\left(\frac{\phi(x)}{a_{2}+\phi(x)}\right) \psi=0, \\
\psi^{\prime}(0)=0, \quad \psi^{\prime}(1)+r \psi(1)=0 .
\end{gathered}
$$

This theorem states that, if the maximum growth rate is small or if the diffusion coefficient is large, then the organism tends to extinction as time becomes large. We also note for reference that $[\mathrm{K}$ ]

$$
\begin{aligned}
& \lambda_{0}=\min _{\psi} \frac{\int_{0}^{1}\left(\psi^{\prime}(x)\right)^{2} d x+r \psi^{2}(1)}{\left[\int_{0}^{1} w_{1}(x) \psi^{2}(x) d x\right]^{1 / 2}}>0, \\
& \mu_{0}=\min _{\psi} \frac{\int_{0}^{1}\left(\psi^{\prime}(x)\right)^{2} d x+r \psi^{2}(1)}{\left[\int_{0}^{1} w_{2}(x) \psi^{2}(x) d x\right]^{1 / 2}}>0,
\end{aligned}
$$


where

$$
w_{i}(x)=\frac{\phi(x)}{a_{i}+\phi(x)}, \quad i=1,2 .
$$

Proof. We prove part (i); (ii) follows similarly. From Lemma 2.1, given $\varepsilon>0$, there exists $t_{0}>0$ such that $S(t, x) \leqq \phi(x)+\varepsilon$ for all $t \geqq t_{0}, 0 \leqq x \leqq 1$. Let $U(t, x)$ be the solution of

$$
\begin{gathered}
\frac{\partial U}{\partial t}=d \frac{\partial^{2} U}{\partial x^{2}}+\frac{m_{1}(\phi(x)+\varepsilon)}{a_{1}+\phi(x)+\varepsilon} U, \\
\frac{\partial U}{\partial x}(t, 0)=0, \quad \frac{\partial U}{\partial x}(t, 1)+r U(t, 1)=0, \\
U\left(t_{0}, x\right)>u\left(t_{0}, x\right), \quad 0 \leqq x \leqq 1 .
\end{gathered}
$$

We show that $u(t, x)<U(t, x)$ for all $t \geqq t_{0}, 0 \leqq x \leqq 1$. Let $w(t, x)=u(t, x)-$ $U(t, x)$. Then, from (2.13) and (3.1), it follows that

$$
\begin{gathered}
d \frac{\partial^{2} w}{\partial x^{2}}-\frac{\partial w}{\partial t}=\frac{m_{1}(\phi(x)+\varepsilon)}{a_{1}+\phi(x)+\varepsilon} U-\frac{m_{1}(\phi(x)-u)}{a_{1}+\phi(x)-u} u \\
\frac{\partial w}{\partial x}(t, 0)=0, \quad \frac{\partial w}{\partial x}(t, 1)+r w(t, 1)=0 \\
w\left(t_{0}, x\right)<0, \quad 0 \leqq x \leqq 1 .
\end{gathered}
$$

The above assertion is equivalent to $w(t, x)<0$ for all $t \geqq t_{0}, 0 \leqq x \leqq 1$.

If not, let $t_{1}$ be the first time for which there is an $x_{1}$ with $w\left(t_{1}, x_{1}\right)=0$. Then, from (3.9) for $0<x<1, t_{0}<t \leqq t_{1}$, it follows that

$$
d \frac{\partial^{2} w}{\partial x^{2}}-\frac{\partial w}{\partial t} \geqq 0
$$

From the maximum principle [PW, p. 160], the maximum of $w$ on $0 \leqq x \leqq 1, t_{0} \leqq t \leqq$ $t_{1}$ must occur along $S_{1}=\left\{t_{0} \leqq t \leqq t_{1}, x=0\right\}$ or $S_{2}=\left\{t_{0} \leqq t \leqq t_{1}, x=1\right\}$ or $S_{3}=$ $\left\{t=t_{0}, 0<x<1\right\}$. Equation (3.11) rules out a nonnegative maximum occurring on $S_{3}$. If $w$ has a nonnegative maximum $w(t, 0)$ on $S_{1}$, then, from the maximum principle [PW, p. 170], it follows that $(\partial w / \partial x)(t, 0)<0$, which contradicts (3.10). Similarly, if $w$ has a nonnegative maximum $w(t, 1)$ on $S_{2}$, then $(\partial w / \partial x)(t, 1)>0$, which contradicts (3.10) since $(\partial w / \partial x)(t, 1)=-\gamma w(t, 1) \leqq 0$.

Next, we establish that $U(x, t) \leqq K e^{-\alpha\left(t-t_{0}\right)}$ for some $K>0, \alpha>0$ and for all $t \geqq$ $t_{0}, 0 \leqq x \leqq 1$. Let $\psi(x)>0,0 \leqq x \leqq 1,\|\psi\|_{2}=1$ be the principal eigenfunction corresponding to the first eigenvalue $\lambda_{0}>0$ of (3.2). Apply "separation of variables" by setting $U(x, t)=z(x, t) \psi(x) e^{-\alpha\left(t-t_{0}\right)}$, where $\alpha>0$ is to be determined. Then $z(x, t)$ satisfies

$$
\begin{gathered}
d \frac{\partial^{2} z}{\partial x^{2}}-\frac{\partial z}{\partial t}+2 d \frac{\psi^{\prime}(x)}{\psi(x)} \frac{\partial z}{\partial x}+\frac{1}{\psi(x)}\left[d \psi^{\prime \prime}+\alpha \psi+\frac{m_{1}(\phi+\varepsilon)}{a_{1}+(\phi+\varepsilon)} \psi\right] z=0 \\
\frac{\partial z}{\partial x}(t, 0)=0, \quad \frac{\partial z}{\partial x}(t, 1)=0 .
\end{gathered}
$$


Consider the square bracket in (3.12). From the assumption $m_{1} / d<\lambda_{0}$ and (3.2) with $\lambda=\lambda_{0}$, it follows that

$$
\begin{aligned}
& d \psi^{\prime \prime}+\alpha \psi+\frac{m_{1}(\phi(x)+\varepsilon)}{a_{1}+\phi(x)+\varepsilon} \psi \\
& =-\frac{d \lambda_{0} \phi(x)}{a_{1}+\phi(x)} \psi+\alpha \psi+\frac{m_{1}(\phi(x)+\varepsilon)}{a_{1}+\phi(x)+\varepsilon} \psi \\
& \quad \leqq \alpha \psi+\left(\frac{m_{1}(\phi(x)+\varepsilon)}{a_{1}+(\phi(x)+\varepsilon)} \psi-\frac{m_{1} \phi(x)}{a_{1}+\phi(x)} \psi\right)+\left(\frac{m_{1} \phi(x)}{a_{1}+\phi(x)} \psi-\frac{d \lambda_{0} \phi(x)}{a_{1}+\phi(x)} \psi\right)<0,
\end{aligned}
$$

provided that $\varepsilon>0, \alpha>0$ are sufficiently small. From (3.12), we have

$$
d \frac{\partial^{2} z}{\partial x^{2}}-\frac{\partial z}{\partial t}+2 d \frac{\psi^{\prime}}{\psi} \frac{\partial z}{\partial x}>0
$$

Equations (3.13), (3.14), and the maximum principle yield

$$
z(x, t) \leqq z\left(x, t_{0}\right) \leqq \sup _{0 \leqq x \leqq 1}\left(\frac{U\left(x, t_{0}\right)}{\psi(x)}\right) .
$$

Hence, it follows that $u(x, t) \leqq U(x, t) \leqq K e^{-\alpha\left(t-t_{0}\right)}$ for some $K>0$. This completes the proof of Theorem 3.1(i).

THEOREM 3.2. If $m_{1}>\lambda_{0} d$ and $u(t, x)$ is the solution of $(3.1)$, then $\lim _{t \rightarrow \infty} u(t$, $x)=\hat{u}(x)$, where $\hat{u}(x)$ is the unique positive steady-state solution of

$$
\begin{gathered}
\frac{\partial u}{\partial t}=d \frac{\partial^{2} u}{\partial x^{2}}+\frac{m_{1}(\phi(x)-u)}{a_{1}+\phi(x)-u} u, \\
\frac{\partial u}{\partial x}(t, 0)=0, \quad \frac{\partial u}{\partial x}(t, 1)+r u(t, 1)=0, \\
u(0, x)=u_{0}(x) \geqq 0, \quad u_{0}(x) \neq 0 .
\end{gathered}
$$

The proof begins with a simple lemma.

LEMMA 3.3. If $m_{1}>\lambda_{0} d$ and $u(t, x)$ is a solution of (3.1), then

$$
\limsup _{t \rightarrow \infty}\|u(t, \cdot)\|_{\infty}>0 .
$$

Proof. If not, then $u(t, x) \rightarrow 0$ as $t \rightarrow \infty$, uniformly on $0 \leqq x \leqq 1$. Then, for any $\varepsilon>0$, there exists $t_{0}>0$ such that $u(t, x)<\varepsilon$ for $t \geqq t_{0}, 0 \leqq x \leqq 1$. Thus $u(t, x)$ satisfies

$$
\frac{\partial u}{\partial t} \geqq d \frac{\partial^{2} u}{\partial x^{2}}+\frac{m_{1}(\phi(x)-\varepsilon)}{a_{1}+\phi(x)-\varepsilon} u .
$$

The maximum principle yields $u(t, x) \geqq U(t, x)$, where $U(t, x)$ satisfies

$$
\begin{gathered}
\frac{\partial U}{\partial t}=d \frac{\partial^{2} U}{\partial x^{2}}+\frac{m_{1}(\phi(x)-\varepsilon)}{a_{1}+\phi(x)-\varepsilon} U, \\
\frac{\partial U}{\partial t}(t, 0)=0, \quad \frac{\partial U}{\partial t}(t, 1)+r U(t, 1)=0, \\
U\left(t_{0}, x\right)<u\left(t_{0}, x\right), \quad 0 \leqq x \leqq 1 .
\end{gathered}
$$


Again, we attempt a separation of variables by setting

$$
U(t, x)=z(t, x) \psi(x) e^{\beta\left(t-t_{0}\right)},
$$

where $\beta>0$ is to be determined. Then $z(t, x)$ satisfies

$$
\begin{gathered}
d \frac{\partial^{2} z}{\partial x^{2}}-\frac{\partial z}{\partial t}+2 d \frac{\psi^{\prime}(x)}{\psi(x)} \frac{\partial z}{\partial x}+\frac{1}{\psi(x)}\left[d \psi^{\prime \prime}-\beta \psi+\frac{m_{1}(\phi-\varepsilon)}{a_{1}+\phi-\varepsilon} \psi\right] z=0 \\
\frac{\partial z}{\partial x}(t, 0)=0, \quad \frac{\partial z}{\partial x}(t, 1)=0 .
\end{gathered}
$$

Since $m_{1} / d>\lambda_{0}$, for small $\varepsilon, \beta>0$, it follows that

$$
\begin{aligned}
d \psi^{\prime \prime}-\beta \psi+\frac{m_{1}(\phi-\varepsilon)}{a_{1}+\phi-\varepsilon} \psi= & \frac{d\left(m_{1} / d-\lambda_{0}\right) \phi(x)}{a_{1}+\phi(x)} \psi-\beta \psi \\
& +\left(\frac{m_{1}(\phi(x)-\varepsilon)}{a_{1}+(\phi(x)-\varepsilon)} \psi-\frac{m_{1} \phi(x)}{a_{1}+\phi(x)} \psi\right)>0,
\end{aligned}
$$

for all $0 \leqq x \leqq 1$. From (3.17), (3.19), we have that

$$
d \frac{\partial^{2} z}{\partial x^{2}}-\frac{\partial z}{\partial t}+2 d \frac{\psi^{\prime}}{\psi} \frac{\partial z}{\partial x}<0 .
$$

Then (3.18), (3.20), and minimum principle yield

$$
z(x, t) \geqq z\left(x, t_{0}\right) \geqq \inf _{0 \leqq x \leqq 1}\left(\frac{U\left(t_{0}, x\right)}{\psi(x)}\right)>0 .
$$

Hence it follows that $u(x, t) \geqq K e^{\beta\left(t-t_{0}\right)}$ for some $K>0$. This contradicts the boundedness of $u(t, x)$ and completes the proof of the lemma.

LEMMA 3.4. If $m_{1}>\lambda_{0} d$, there exists a unique positive steady-state solution of $\hat{u}(x)$ of (3.15) and $\lim _{t \rightarrow \infty} u(t, x)=\hat{u}(x)$.

Proof. Any steady state of (3.15) satisfies

$$
\begin{gathered}
d u^{\prime \prime}+\frac{m_{1}(\phi-u)}{a_{1}+\phi-u} u=0, \\
u^{\prime}(0)=0, \quad u^{\prime}(1)+r u(1)=0 .
\end{gathered}
$$

In [SoW] the authors proved that, when $m_{1}$ is considered as a bifurcation parameter, the bifurcation occurs at $m_{1}=\lambda_{0} d$, and for $m_{1} / d>\lambda_{0}$ there exists a positive solution $\hat{u}(x)$ of (3.22), (3.23) satisfying $0<\hat{u}(x)<\phi(x)$. Suppose that $u_{1}(x), u_{2}(x)$ were two positive steady states satisfying $0<u_{1}(x), u_{2}(x)<\phi(x)$ for $0 \leqq x \leqq 1$. Then we have the following two cases.

Case 1. The curves $y=u_{1}(x)$ and $y=u_{2}(x)$ do not intersect on $0 \leqq x \leqq 1$. Without loss of generality, assume that $u_{1}(x)<u_{2}(x)$ for all $0 \leqq x \leqq 1$. Let $\omega=u_{2} / u_{1}$. Then it follows that

$$
\begin{gathered}
\omega^{\prime \prime}+2 \omega^{\prime} \frac{u_{1}^{\prime}}{u_{1}}+\left(\frac{1}{d}\right) c(x) \omega=0, \\
\omega^{\prime}(0)=0, \quad \omega^{\prime}(1)=0,
\end{gathered}
$$


where

$$
c(x)=\frac{m_{1}\left(\phi-u_{2}\right)}{a_{1}+\phi-u_{2}}-\frac{m_{1}\left(\phi-u_{1}\right)}{a_{1}+\phi-u_{1}} \leqq 0 .
$$

From (3.25) and maximum principle, $\omega(x) \equiv$ constant. Since $0<u_{1}(x)<u_{2}(x)$ for all $x \in[0,1], \omega(x) \equiv 0$. This contradicts the fact that $\omega=u_{2} / u_{1}>0$ for all $0 \leqq x \leqq 1$.

Case 2. The curves $y=u_{1}(x)$ and $y=u_{2}(x)$ intersect at some point. Without loss of generality, from $(3.23), u_{i}^{\prime}(0)=0, i=1,2$, and the uniqueness of solutions of ordinary differential equations, we may assume that $u_{1}(0)<u_{2}(0)$ and $u_{1}(x)<u_{2}(x)$ on $(0, \delta)$ for some $0<\delta<1, u_{1}(\delta)=u_{2}(\delta)$. Let $\omega=u_{2} / u_{1}$. Then $\omega(x)$ satisfies (3.24)-(3.26) and $\omega(0)>1$. From the maximum principle, the maximum of $\omega(x)$ on $[0, \delta]$ occurs at $x=\delta$. Hence $\omega(\delta)>\omega(0)>1$ or $u_{2}(\delta)>u_{1}(\delta)$, which is a contradiction.

It remains to show the convergence claimed in the lemma. Consider the Lyapunov functional [H, p. 79]

$$
V(\psi)=\int_{0}^{1}\left[\frac{d}{2}\left(\frac{\partial \psi}{\partial x}\right)^{2}-F(x, \psi(x))\right] d x+B(\psi),
$$

where

$$
F(x, u)=\int_{0}^{u} f(x, s) d s, \quad f(x, u)=\frac{m_{1}(\phi(x)-u)}{a_{1}+\phi(x)-u} u
$$

and

$$
B(\psi)=\frac{r d}{2} \psi^{2}(1)
$$

Then it follows that

$$
\frac{d}{d t} V(u(t, x))=-\int_{0}^{1}\left(\frac{\partial u}{\partial t}\right)^{2} d x \leqq 0
$$

and $u(x, t)$ approaches the largest invariant set where $\int_{0}^{1}(\partial u / \partial t)^{2} d x=0$, that is, the set of steady states. Since $m_{1} / d>\lambda_{0}$, from Lemma 3.3, the only positive steady state is $\hat{u}$. This completes the proof of Theorem 3.2.

Similar results hold for the $v$ population. If we label the $v$ equation with a prime, as we do with the following variation on $\left(3.1^{\prime}\right)$ :

$$
\begin{gathered}
\frac{\partial v}{\partial t}=d \frac{\partial^{2} v}{\partial x^{2}}+\frac{m_{2}(\phi(x)-v)}{a_{2}+\phi(x)-v} v=0, \\
\frac{\partial v}{\partial x}(t, 0)=0, \quad \frac{\partial v}{\partial x}(t, 1)+r v(t, x)=0,
\end{gathered}
$$

then a similar theorem holds for $v(t, x)$, where $\mu_{0}$ replaces $\lambda_{0}$. We refer to these results as Theorem $3.2^{\prime}$. We can now easily obtain the following extinction result.

THEOREM 3.5. Let $u(t, x)$ and $v(t, x)$ be solutions of (2.13)-(2.15). (i) If $m_{1}>$ $\lambda_{0} d$ and $m_{2}<\mu_{0} d$, then $\lim _{t \rightarrow \infty} u(t, x)=\hat{u}(x)$ and $\lim _{t \rightarrow \infty} v(t, x)=0$ uniformly for 
$0 \leqq x \leqq 1$, where $\hat{u}(x)$ is the unique positive steady state of

$$
\begin{gathered}
\frac{\partial u}{\partial t}=d \frac{\partial^{2} u}{\partial x^{2}}+\frac{m_{1}(\phi(x)-u)}{a_{1}+(\phi(x)-u)} u, \\
\frac{\partial u}{\partial x}(t, 0)=0, \quad \frac{\partial u}{\partial x}(t, 1)+r u(t, 1)=0, \\
u(0, x)=u_{0}(x) \geqq 0, \quad u_{0}(x) \supsetneqq 0 .
\end{gathered}
$$

(ii) If $m_{1}<\lambda_{0} d$ and $m_{2}>\mu_{0} d$, then $\lim _{t \rightarrow \infty} u(t, x)=0$ and $\lim _{t \rightarrow \infty} v(t, x)=$ $\tilde{v}(x)$ uniformly for $0 \leqq x \leqq 1$, where $\tilde{v}(x)$ is the unique positive steady state of

$$
\begin{gathered}
\frac{\partial v}{\partial t}=d \frac{\partial^{2} v}{\partial x^{2}}+\frac{m_{2}(\phi(x)-v)}{a_{2}+\phi(x)-v} v, \\
\frac{\partial v}{\partial x}(t, 0)=0, \quad \frac{\partial v}{\partial x}(t, 1)+r v(t, 1)=0, \\
v(0, x)=v_{0}(x) \geqq 0, \quad v_{0}(x) \neq 0 .
\end{gathered}
$$

Proof. We prove part (i). Part (ii) follows similarly. Let $u(t, x), v(t, x)$ be solutions of (2.13)-(2.15). Then $v(t, x)$ satisfies

$$
\begin{gathered}
\frac{\partial v}{\partial t} \leqq d \frac{\partial^{2} v}{\partial x^{2}}+f_{2}(\phi-v) v, \\
\frac{\partial v}{\partial x}(t, 0)=0, \quad \frac{\partial v}{\partial x}(t, 1)+r v(t, 1)=0, \\
v(0, x)=v_{0}(x) .
\end{gathered}
$$

Compare the solution of this inequality to $V(t, x)$, a solution of $\left(3.1^{\prime}\right)$ where $v_{0}(x) \leqq$ $v(0, x)$. It follows that $v(t, x) \leqq V(t, x)$. From Theorem 3.1(ii), $\lim _{t \rightarrow \infty} v(t, x)=$ $O\left(e^{-\alpha t}\right)$. Consider the dynamical system defined by (2.13)-(2.15) on $C_{+} \times C_{+}$. The omega limit set lies in $C_{+} \times\{0\}$. Any solution of (2.13)-(2.15) with $v_{0}(x) \equiv 0$, $u_{0}(x) \geqq 0, u_{0}(x) \not 0$, has $u(t, x)$ as a solution of (3.1). Since $m_{1}>\lambda_{0} d$, all such trajectories have $(\hat{u}, 0)$ as their omega limit set. This completes the proof.

The following theorem restates the results of this section in terms of the original system (1.1)-(1.3).

THEOREM 3.6. If $m_{1}<\lambda_{0} d$ and $m_{2}<\mu_{0} d$,

$$
\lim _{t \rightarrow \infty} S(t, x)=\phi(x), \quad \lim _{t \rightarrow \infty} u(t, x)=0, \quad \lim _{t \rightarrow \infty} v(t, x)=0 .
$$

If $m_{1}<\lambda_{0} d$ and $m_{2}>\mu_{0} d$,

(3.27) $\lim _{t \rightarrow \infty} S(t, x)=\phi(x)-\tilde{v}(x), \quad \lim _{t \rightarrow \infty} u(t, x)=0, \quad \lim _{t \rightarrow \infty} v(t, x)=\hat{v}(x)$.

If $m_{1}>\lambda_{0} d$ and $m_{2}<\mu_{0} \delta$,

(3.28) $\lim _{t \rightarrow \infty} S(t, x)=\phi(x)-\hat{u}(x), \quad \lim _{t \rightarrow \infty} u(t, x)=\hat{u}(x), \quad \lim _{t \rightarrow \infty} v(t, x)=0$.

Thus, to have an interesting problem (i.e., to have meaningful competition), we make the basic hypothesis

$$
m_{1}>d \lambda_{0}, \quad m_{2}>d \mu_{0}
$$


Under hypothesis $(\mathrm{H})$, each competitor would survive in the chemostat without competition. In the following, an extinction result under the hypothesis $(\mathrm{H})$ is established.

THEOREM 3.7. Let $(\mathrm{H})$ hold. (i) If

$$
m_{2} \leqq m_{1}, \quad \frac{m_{2}}{m_{1}}<\frac{a_{2}}{a_{1}}
$$

or

$$
m_{2}>m_{1}, \quad \frac{m_{2}}{m_{1}}<\frac{a_{2}}{a_{1}}, \quad 0<\phi(x)<\frac{m_{1} a_{2}-m_{2} a_{1}}{m_{2}-m_{1}},
$$

then (3.28) holds. (ii) If

$$
m_{1} \leqq m_{2}, \quad \frac{m_{1}}{m_{2}}<\frac{a_{1}}{a_{2}}
$$

or

$$
m_{1}>m_{2}, \quad \frac{m_{1}}{m_{2}}<\frac{a_{1}}{a_{2}}, \quad 0<\phi(x)<\frac{m_{2} a_{1}-m_{1} a_{2}}{m_{1}-m_{2}},
$$

then (3.27) holds.

Before we prove Theorem 3.7, we begin with the following technical lemma.

LEMMA 3.8. There exists $C_{0}>0$ such that the solution $S(t, x)$ of $(1.1)-(1.3)$ satisfies $S(t, x) \geqq C_{0}>0$ for $t \geqq t_{0}, 0 \leqq x \leqq 1$ for $t_{0}$ sufficiently large.

Proof. From (2.1) and the first equation of (1.1), given $\varepsilon>0$, there exists $T>0$ such that for all $t \geqq T, 0 \leqq x \leqq 1$, we have

$$
\frac{\partial S}{\partial t} \geqq d \frac{\partial^{2} S}{\partial x^{2}}-c S(\phi(x)+\varepsilon-S)
$$

or

$$
\frac{\partial S}{\partial t} \geqq d \frac{\partial^{2} S}{\partial x^{2}}-c S(K-S),
$$

where

$$
c=\max \left(\frac{m_{1}}{a_{1}}, \frac{m_{2}}{a_{2}}\right) \text { and } K>2\|\phi\|_{\infty} .
$$

Comparing the solution $S(t, x)$ with the solution $z(t, x)$ of

$$
\begin{gathered}
\frac{\partial z}{\partial t}=d \frac{\partial^{2} z}{\partial x^{2}}-c z(K-z), \\
\frac{\partial z}{\partial x}(t, 0)=-S^{(0)}, \quad \frac{\partial z}{\partial x}(t, 1)+r z(t, 1)=0, \\
0<z(T, x)<S(T, x),
\end{gathered}
$$

yields $S(t, x) \geqq z(t, x), t \geqq T, 0 \leqq x \leqq 1$. The solution $z(t, x)$ is positive, and converges to the set of steady states of (3.29), (3.30) [H2]. We show that (3.29), (3.30) has a unique positive steady state, and hence $z(t, x)$ converges to the unique positive steady state $z^{*}(x)$. Let $C_{0}$ satisfy $0<C_{0}<\frac{1}{2} \min _{0 \leqq x \leqq 1} z^{*}(x)$. Then, for $t$ sufficiently large, it follows that $S(t, x) \geqq z(t, x) \geqq C_{0}$, and the proof of the lemma is complete. 
Let $z_{1}(x)$ and $z_{2}(x)$ be two positive solutions of

$$
d z^{\prime \prime}-c z(K-z)=0, \quad z^{\prime}(0)=-S^{(0)}, \quad z^{\prime}(1)+r z(1)=0 .
$$

Without loss of generality, there are two possible cases: (i) $z_{1}(x)<z_{2}(x)$ for all $0 \leqq x \leqq$ 1, and (ii) $z_{1}(x)$ and $z_{2}(x)$ intersects transversally at some points. For either case, we may assume that $z_{1}(x)<z_{2}(x)$ on $(\delta, 1)$, where $\delta$ satisfies $\delta=0, z_{1}(\delta)<z_{2}(\delta)$, $z_{1}^{\prime}(\delta)=z_{2}^{\prime}(\delta)=-S^{(0)}$ in case (i), while, for case (ii), $\delta>0, z_{1}(\delta)=z_{2}(\delta), z_{2}^{\prime}(\delta) \geqq$ $z_{1}^{\prime}(\delta)$. Integrating (3.35) from $\delta$ to 1 yields

$$
z_{i}^{\prime}(1)=z_{i}^{\prime}(\delta)+\frac{c}{d} \int_{\delta}^{1} z_{i}\left(K-z_{i}\right) d x
$$

and

$$
z_{i}(1)=z_{i}(\delta)+z_{i}^{\prime}(\delta)(1-\delta)+\frac{c}{d} \int_{\delta}^{1} \int_{\delta}^{x} z_{i}\left(K-z_{i}\right) d \xi d x
$$

for $i=1$, 2. Since $\left\|z_{i}\right\|_{\infty} \leqq\|\phi\|_{\infty} \leqq K / 2$, it follows that $z_{2}(x)\left(K-z_{2}(x)\right)>$ $z_{1}(x)\left(K-z_{1}(x)\right)$ for $\delta<x \leqq 1$. Then it holds that

$$
\begin{aligned}
0= & z_{2}^{\prime}(1)+r z_{2}(1) \\
= & z_{2}^{\prime}(\delta)+\frac{c}{d} \int_{\delta}^{1} z_{2}\left(K-z_{2}\right) d x+r z_{2}(\delta) \\
& +r z_{2}^{\prime}(\delta)(1-\delta)+r \frac{c}{d} \int_{\delta}^{1} \int_{\delta}^{x} z_{2}\left(K-z_{2}\right) d \xi d x \\
> & z_{1}^{\prime}(\delta)+\frac{c}{d} \int_{\delta}^{1} z_{1}\left(K-z_{1}\right) d x+r z_{1}(\delta) \\
& +r z_{1}^{\prime}(\delta)(1-\delta)+r \frac{c}{d} \int_{\delta}^{1} \int_{\delta}^{x} z_{1}\left(K-z_{1}\right) d \xi d x \\
= & z_{1}^{\prime}(1)+r z_{1}(1)=0 .
\end{aligned}
$$

This is the desired contradiction.

Proof of Theorem 3.7. Proof is given for Theorem 3.7(i); part (ii) follows by the same argument. Let $\omega=v / u$. Then $\omega(t, x)$ satisfies

$$
\begin{gathered}
d \frac{\partial^{2} \omega}{\partial x^{2}}-\frac{\partial \omega}{\partial t}+2 d\left(\frac{\partial u}{\partial x}\right)\left(\frac{1}{u}\right) \frac{\partial \omega}{\partial x}+c(x, t) \omega=0, \\
\frac{\partial \omega}{\partial x}(t, 0)=0, \quad \frac{\partial \omega}{\partial x}(t, 1)=0
\end{gathered}
$$

where

$$
\begin{aligned}
c(x, t) & =\frac{m_{2} S(t, x)}{a_{2}+S(t, x)}-\frac{m_{1} S(t, x)}{a_{1}+S(t, x)} \\
& =\frac{S}{\left(a_{1}+S\right)\left(a_{2}+S\right)}\left[\left(m_{2}-m_{1}\right) S+\left(m_{2} a_{1}-m_{1} a_{2}\right)\right] .
\end{aligned}
$$

From the assumptions in (i) and Lemma 3.8, there exists $\delta>0, c(x, t) \leqq-\delta$ for $t \geqq t_{0}, 0 \leqq x \leqq 1$. We show that $\omega(x, t)$ decays to zero exponentially. Let

$$
\omega(t, x)=z(t, x) e^{-\beta\left(t-t_{0}\right)},
$$


where $\beta>0$ is to be determined. By substituting into (3.33), (3.34), we see that $z(t, x)$ satisfies

$$
\begin{gathered}
d \frac{\partial^{2} z}{\partial x^{2}}-\frac{\partial z}{\partial t}+2 d\left(\frac{\partial u}{\partial x}\right)\left(\frac{1}{u}\right) \frac{\partial z}{\partial x}+(\beta+c(x, t)) z=0, \\
\frac{\partial z}{\partial x}(t, 0)=0, \quad \frac{\partial z}{\partial x}(t, 1)=0 .
\end{gathered}
$$

Let $0<\beta<\delta$. Then $\beta+c(x, t)<0$ for all $t \geqq t_{0}, 0 \leqq x \leqq 1$. From the maximum principle, it follows that $z(x, t) \leqq z\left(x, t_{0}\right), t \geqq t_{0}, 0 \leqq x \leqq 1$, and hence that

$$
v(x, t)=u(x, t) z\left(x, t_{0}\right) e^{-\beta\left(t-t_{0}\right)} \leqq M e^{-\beta t}
$$

for some $M>0$. Thus $v(x, t)$ decays to zero exponentially. Then the same dynamical systems arguments used in Theorem 3.5(i) complete the proof of the theorem.

4. Coexistence. As noted above, on a set contained in the attractor, the semidynamical system satisfies

$$
\begin{gathered}
\frac{\partial u}{\partial t}=d \frac{\partial^{2} u}{\partial x^{2}}+f_{1}(\phi-u-v) u, \\
\frac{\partial v}{\partial t}=d \frac{\partial^{2} v}{\partial x^{2}}+f_{2}(\phi-u-v) v, \\
\frac{\partial u}{\partial x}(t, 0)=\frac{\partial v}{\partial x}(t, 0)=0, \\
\frac{\partial u}{\partial x}(t, 1)+r u(t, 1)=0, \quad \frac{\partial v}{\partial x}(t, 1)+r v(t, 1)=0, \\
u(0, x)=u_{0}(x) \geqq 0, \quad v(0, x)=v_{0}(x) \geqq 0, \\
u_{0}(x) \geqq 0, \quad v_{0}(x) \neq 0, \\
\phi(x)-u_{0}(x)-v_{0}(x) \geqq 0, \\
f_{i}(S)= \begin{cases}m_{i} S /\left(a_{i}+S\right), & S \geqq 0, \\
0, & S \leqq 0 .\end{cases}
\end{gathered}
$$

We first determine the stability of the rest points. Linearize about $(\hat{u}, 0)$ to obtain

$$
\begin{gathered}
\frac{\partial u}{\partial t}=d \frac{\partial^{2} u}{\partial x^{2}}+\left[\frac{-m_{1} a_{1} \hat{u}}{\left(a_{1}+\phi-\hat{u}\right)^{2}}+\frac{m_{1}(\phi-\hat{u})}{\left(a_{1}+\phi-\hat{u}\right)}\right] u+\left[\frac{-m_{1} a_{1} \hat{u}}{\left(a_{1}+\phi-\hat{u}\right)^{2}}\right] v, \\
\frac{\partial v}{\partial t}=d \frac{\partial^{2} v}{\partial x^{2}}+\frac{m_{2}(\phi-\hat{u})}{a_{2}+\phi-\hat{u}} v, \\
\frac{\partial u}{\partial x}(t, 0)=\frac{\partial v}{\partial x}(t, 0)=0, \\
\frac{\partial u(t, 1)}{\partial x}+r u(t, 1)=0, \\
\frac{\partial v(t, 1)}{\partial x}+r v(t, 1)=0 .
\end{gathered}
$$


Let $u(t, x)=p(x) e^{\lambda t}, v(t, x)=q(x) e^{\lambda t}$. Then we have that

$$
\begin{gathered}
\lambda p(x)=d p^{\prime \prime}+\left[\frac{-m_{1} a_{1} \hat{u}}{\left(a_{1}+\phi-\hat{u}\right)^{2}}+\frac{m_{1}(\phi-\hat{u})}{a_{1}+\phi-\hat{u}}\right] p+\frac{-m_{1} a_{1} \hat{u}}{\left(a_{1}+\phi-\hat{u}\right)^{2}} q, \\
\lambda q(x)=d q^{\prime \prime}+\frac{m_{2}(\phi-\hat{u})}{a_{2}+\phi-\hat{u}} q, \\
p^{\prime}(0)=0, \quad q^{\prime}(0)=0, \\
p^{\prime}(1)+r p(1)=0, \quad q^{\prime}(1)+r q(1)=0 .
\end{gathered}
$$

Since (4.1) is a competitive system [S], from [P], it follows that the eigenvalue with the largest real part is real. Hence it is sufficient to consider the second equation, which is independent of $\mathrm{p}$.

Think of $m_{2}$ as a parameter and let $\hat{\lambda}\left(m_{2}\right)$ be the largest eigenvalue of the above Sturm-Liouville problem. (Note that the sign of eigenvalue is opposite the Sturm-Liouville problem, as found in [CL], so that the largest is correct.) From [K], we have

$$
\hat{\lambda}\left(m_{2}\right)=\max _{q>0}\left[\frac{-d r q^{2}(1)-d \int_{0}^{1}\left(q^{\prime}(x)\right)^{2} d x+\int_{0}^{1} m_{2} \hat{S}(x) /\left(a_{2}+\hat{S}(x)\right) q^{2}(x) d x}{\int_{0}^{1} q^{2}(x) d x}\right] .
$$

From (4.2), $\hat{\lambda}\left(m_{2}\right)$ is a strictly increasing function of $m_{2}$ satisfying $\hat{\lambda}\left(m_{2}\right)<0$ if $m_{2}$ is small and $\hat{\lambda}\left(m_{2}\right) \rightarrow+\infty$ as $m_{2} \rightarrow+\infty$. Since $\hat{\lambda}\left(m_{2}\right)$ is monotone; there is a unique value $m_{2}^{*}$ such that $\hat{\lambda}\left(m_{2}^{*}\right)=0$. If $m_{2}>m_{2}^{*}$, the largest eigenvalue is positive, and $\hat{E}$ is unstable. There is a similar result for $\tilde{E}$. Table 1 describes the situation.

THEOREM 4.1. Fix $m_{1}$ and $m_{2}$ so that $(\mathrm{H})$ holds. If $m_{1}>m_{1}^{*}$ and $m_{2}>m_{2}^{*}$, then the semidynamical system generated by (4.1) is uniformly persistent.

Proof. We seek to apply the general persistence theorem in [HW]. Undefined terms are taken from that paper. As noted previously, (4.1) generates a semidynamical system $T(t) x$ on $C_{+}[0,1] \times C_{+}[0,1] . T$ is compact for $t>0$, and it has already been noted that $T(t) x$ is dissipative. Let

$$
X^{0}=\left\{(u, v) \in C^{+}[0,1] \times C^{\times}[0,1], u(x)>0 \text { and } v(y)>0 \text {, some } x, y \in(0,1)\right\} .
$$

$X^{0}$ is open and invariant, and $\partial X^{0}$ is invariant. Let $X=\overline{X^{0}}$. Since $\hat{E}$ attracts $(u, 0)$, $u \not \equiv, u \geqq 0$ and $\tilde{E}$ attracts $(0, v), v \not \equiv 0, v \geqq 0$, the omega limit sets of the semidynamical system on the boundary, denoted (as in [HW]) by $\tilde{A}_{\delta}$, are given by $\tilde{A}_{\delta}=\left\{E_{0}, \hat{E}, \tilde{E}\right\}$. Let $M=\left\{M_{1}, M_{2}, M_{3}\right\}=\left\{E_{0}, \hat{E}, \tilde{E}\right\}$ be a covering of $\tilde{A}_{\delta}$ as defined in [HW]. Since the origin is repeller, there are no cycles [HW] in the boundary. It remains to check that the stable sets of $W^{+}\left(M_{i}\right)$ do not intersect $\bar{X}^{0}$; that is, $W^{+}\left(M_{i}\right) \cap X^{0}=\varnothing$ and that the covering is an isolated covering. Essentially the same argument is used for both these facts.

TABLE 1

\begin{tabular}{cccc}
\hline & Exists & Unstable & Stable \\
\hline$E_{0}=(0,0)$ & Always & Always & Never \\
$\hat{E}=(\hat{u}, 0)$ & $m_{1}>\lambda_{0} d$ & $m_{2}>m_{2}^{*}$ & $m_{2}<\mu_{0} d$ \\
$\tilde{E}=(0, \tilde{v})$ & $m_{2}>\mu_{0} d$ & $m_{1}>m_{1}^{*}$ & $m_{1}<\lambda_{0} d$ \\
\hline
\end{tabular}


Suppose that there exists $\left(u_{0}, v_{0}\right)$ in the stable set of $(\hat{u}, 0)$ with $u_{0}>0, v_{0}>0$. Let $u(x, t)$ and $v(x, t)$ be the (necessarily positive) solutions with these initial conditions satisfying $\lim _{t \rightarrow \infty} u(\cdot, t)=\hat{u}(\cdot)$ and $\lim _{t \rightarrow \infty} v(\cdot, t)=0$. For $\varepsilon>0$, there is a $t_{0}>0$ such that, for $t \geqq t_{0}$,

$$
u(x, t)+v(x, t) \leqq \hat{u}(x)+\varepsilon .
$$

Hence $v(x, t)$ satisfies

$$
\frac{\partial v}{\partial t}=d \frac{\partial^{2} v}{\partial x^{2}}+\frac{m_{2}(\phi-u-v)}{a_{2}+(\phi(x)-u-v)} v \geqq d \frac{\partial^{2} v}{\partial x^{2}}+\frac{m_{2}(\phi-\hat{u}(x)-\varepsilon)}{a_{2}+(\phi(x)-\hat{u}(x)-\varepsilon)} v .
$$

Comparing this solution with the corresponding solution of

$$
\begin{gathered}
\frac{\partial V}{\partial t}=d \frac{\partial^{2} V}{\partial x^{2}}+\frac{\left.m_{2}(\phi(x)-\hat{u}+\varepsilon)\right)}{a_{2}+\phi(x)-\hat{u}-\varepsilon} V, \\
\frac{\partial V}{\partial t}(t, 0)=0, \quad \frac{\partial V}{\partial t}(t, 1)+r V(t, 1)=0, \\
0<V\left(t_{0}, x\right)<v\left(t_{0}, x\right)
\end{gathered}
$$

allows us to conclude that $0<V(t, x)<v(t, x)$. We seek to solve the linear equation by separation of variables. Let

$$
V(x, t)=z(x, t) \Psi(x) e^{\beta\left(t-t_{0}\right)},
$$

where $\beta$ is to be determined and $\Psi(x)>0$ is the principal eigenfunction corresponding to the largest eigenvalue $\hat{\lambda}\left(m_{2}\right)$ of

$$
\begin{aligned}
& \lambda q=d q^{\prime \prime}+\left(\frac{m_{2}(\phi(x)-\hat{u}(x))}{a_{2}+\phi(x)-\hat{u}(x)}\right) q, \\
& q^{\prime}(0)=0, \quad q^{\prime}(1)+r q(1)=0 .
\end{aligned}
$$

Recall that this is real and that the eigenfunction is positive for $m_{2}$ large enough. A computation yields that $z(x, t)$ satisfies

$$
\begin{gathered}
d \frac{\partial^{2} z}{\partial x^{2}}-\frac{\partial z}{\partial t}+2 d \frac{\Psi^{\prime}(x)}{\Psi(x)} \frac{\partial z}{\partial x}+\frac{1}{\Psi(x)}\left[d \Psi^{\prime \prime}-\beta \Psi+\frac{m_{2}(\hat{S}-\varepsilon)}{a_{2}+\hat{S}-\varepsilon} \Psi\right] z=0 \\
\frac{\partial z}{\partial x}(t, 0)=0, \quad \frac{\partial z}{\partial t}(t, 1)=0
\end{gathered}
$$

We estimate the term in the square brackets by

$$
\begin{aligned}
& d \Psi^{\prime \prime}-\beta \Psi+\frac{m_{2}(\phi(x)-\hat{u}(x)-\varepsilon)}{a_{2}+\phi(x)-\hat{u}(x)-\varepsilon} \Psi \\
& =\left(d \Psi^{\prime \prime}+\frac{m_{2}(\phi(x)-\hat{u}(x))}{a_{2}+\phi(x)-\hat{u}(x)} \Psi\right)-\beta \Psi+\left(\frac{m_{2}(\phi(x)-\hat{u}(x)-\varepsilon)}{a_{2}+\phi(x)-\hat{u}(x)-\varepsilon}-\frac{m_{2} \phi(x)-\hat{u}(x)}{a_{2}+\phi(x)-\hat{u}(x)}\right) \Psi \\
& =\hat{\lambda}\left(m_{2}\right) \Psi-\beta \Psi+\left(\frac{m_{2}(\phi(x)-\hat{u}(x)-\varepsilon)}{a_{2}+\phi(x)-\hat{u}(x)-\varepsilon}-\frac{m_{2} \phi(x)-\hat{u}(x)}{a_{2}+\phi(x)-\hat{u}(x)}\right) \Psi \\
& =\left[\hat{\lambda}\left(m_{2}\right)-\beta+\left(\frac{m_{2}(\phi(x)-\hat{u}(x)-\varepsilon)}{a_{2}+\phi(x)-\hat{u}(x)-\varepsilon}-\frac{m_{2}(\phi(x)-\hat{u}(x))}{a_{2}+\phi(x)-\hat{u}(x)}\right)\right] \Psi>0,
\end{aligned}
$$


provided that $\beta>0$ and $\varepsilon>0$ are chosen sufficiently small. Then

$$
d \frac{\partial^{2} z}{\partial x^{2}}-\frac{\partial z}{\partial t}+2 d \frac{\Psi^{\prime}(x)}{\Psi(x)} \frac{\partial z}{\partial x}<0 .
$$

Thus the minimum principle implies that (note Neumann boundary conditions)

$$
z(x, t) \geqq z\left(x, t_{0}\right) \geqq \inf _{0 \leqq x \leqq 1} \frac{V\left(x, t_{0}\right)}{\Psi(x)}=C>0
$$

and hence that $v(x, t) \geqq V(x, t) \geqq C \Psi(x) e^{\beta\left(t-t_{0}\right)} \geqq K e^{\beta\left(t-t_{0}\right)}$. This contradicts the assumption that $\lim _{t \rightarrow \infty} v(x, t)=0$.

If one of the rest points is not isolated, then a trajectory must remain in every neighborhood of that rest point, say, in a neighborhood of $\hat{E}$. Then inequality (4.3) must be satisfied for every $\varepsilon>0$ and some trajectory. The same estimates apply. Hale and Waltmon [HW, Thm. 4.1] complete the proof of uniform persistence.

From [HW, Thm. 3.2], there are global attractors, $A_{0}$ in $X^{0}, A_{\delta}$ in $\partial X$, and $A$ in $\bar{X}$ and

$$
A=A_{0} \cup W^{-}\left(A_{\delta}\right),
$$

where $W^{-}\left(A_{\delta}\right)$ consists of orbits with alpha limit sets in $A_{\delta}$. If we introduce an order by $\left(u_{1}, v_{1}\right)<_{K}\left(u_{2}, v_{2}\right)$ if $u_{1} \geqq u_{2}, v_{1} \leqq v_{2}$, then $T(t) x$ is a monotone semidynamical system. Using the results of Hirsch [Hi] and Matano [M1], $A_{0}$ must contain a stable rest point, and, if it contains only one, must be identical to it. The foregoing discussion is summarized in the following statement.

THEOREM 4.2. If $(\mathrm{H})$ holds and $m_{1}>m_{1}^{*}, m_{2}>m_{2}^{*}$, there exists at least one positive, stable equilibrium of $(4.1),\left(u_{c}(\cdot), v_{c}(\cdot)\right)$. If there is only one equilibrium, then $\lim _{t \rightarrow \infty}(u(t, \cdot), v(t, \cdot))=\left(u_{c}(\cdot), v_{c}(\cdot)\right)$.

Theorems 4.1 and 4.2 apply to the reduced system (4.1). It is natural to ask about the full system given by (1.1)-(1.3). Theorem 4.2 applies directly, since any fixed point must lie in the set given by $S+u+v-\phi=0$. The points of $A_{0}$ (given by the abovecited decomposition ) in $C_{+} \times C_{+}$can be viewed as points in $C_{+} \times C_{+} \times C_{+}$of the form $\Gamma=(\phi-u-v, u, v)$. By Lemma 3.8, $\phi-u-v \geqq C_{0}>0$. Hence we can take an epsilon neighborhood about $\Gamma$ that does not intersect the boundary of $C_{+} \times C_{+} \times C_{+}$. All trajectories with positive initial conditions are eventually in this set, so that system (1.1)-(1.3) is uniformly persistent.

5. Discussion. We have analyzed a model of the chemostat without the assumption that the vessel is well mixed. The principal result is that removing this hypothesis can lead to coexistence of competing populations in contrast to the competitive exclusion that holds in the basic chemostat. This is biologically important in that it may offer an explanation for coexistence under exploitative competition. The model took the form of a system of reaction-diffusion equations, and recent results on uniform persistence for infinite-dimensional systems [HW] played a prominent role. The results also provided stability results in terms of the PDEs in contrast to the previous work [SoW]. When there is a unique steady state in the interior, coexistence solutions converge to it. The conjecture is that there is at most one interior equilibrium. This would be of interest even in the $n$-vessel gradostat.

A major disadvantage of the model is the assumption of equal diffusion rates. It is not clear whether this makes a difference in the asymptotic behavior, but it is certainly necessary for the approach here. Handling different diffusion rates remains an open question, one worthy of (and presently under) further study. 
In the case of the gradostat [JSTW], [STW], more detailed information was available, and a more complete classification was presented. There is a major untreated gap in this presentation where $\hat{E}, \tilde{E}$ might both be stable $\left(\lambda_{0} d<m_{1}<m_{1}^{*}, \mu_{0} d<m_{2}<m_{2}^{*}\right)$, or one is stable and one is unstable. We conjecture that the first case cannot occur and that, in the second, the locally stable rest point is globally stable. There is hope of answering both if we could establish that a necessary and sufficient condition for the existence of a rest point is that $\hat{E}$ and $\tilde{E}$ are both unstable.

Acknowledgment. Sze-Bi Hsu thanks the Department of Mathematics and Computer Science, Emory University, for the support and hospitality during his 1991-1992 visit, when a portion of this work was accomplished.

\section{REFERENCES}

[CCS] K. N. CHeuh, C. C. CONLEY, AND J. A. SMOlLeR, Positively invariant regions for systems of nonlinear diffusion equations, Indiana Univ. Math. J., 26 (1977), pp. 373-392.

[CL] E. A. Coddington AND N. Levinson, Theory of Ordinary Differential Equations, McGraw-Hill, New York, 1955.

[DRS] S. DUNBAR, K. RYBAKOWSKI, AND K. SCHMITT, Persistence in predator-prey models with diffusion, J. Differential Equations, 65 (1986), pp. 117-138.

[FS] A. G. Fredrickson AND G. StePhanopoulos, Microbial competition, Science, 213 (1981), pp. 972-979.

[H] J. HalE, Asymptotic Behavior of Dissipative Systems, American Mathematical Society, Providence, RI, 1988.

[HET] D. Herbert, R. Elsworth, AND R. C. Telling, The continuous culture of bacteria: A theoretical and experimental study, J. General Microbiology, 14 (1956), pp. 601-622.

[HH] S. R. HANSEN AND S. P. HubBell, Single nutrient microbial competition; Agreement between experimental and theoretical forecast outcomes, Science, 20 (1980), pp. 1491-1493.

[HHW] S. B. HSU, S. P. HubBell, AND P. WALTMAN, A mathematical theory for single nutrient competition in continuous cultures of microorganisms, SIAM J. Appl. Math., 32 (1977), pp. 366-383.

[Hi] M. W. HIRSCH, The dynamical system approach to differential equations, Bull. Amer. Math. Soc., 11 (1984), pp. 1-64.

[HS] V. HutSON AND K. SCHMITT, Permanence in dynamical systems, Math. Biosci., 111 (1992), pp. 171.

[Hsu] S. B. Hsu, Limiting behaviour for competing species, SIAM J. Appl. Math., 34 ( 1978), pp. 760-763.

[HW] J. Hale AND P. Waltman, Persistence in infinite-dimensional systems, SIAM J. Math. Anal., 20 (1989), pp. 388-395.

[JM] H. W. JANNASH AND R. T. MATELES, Experimental bacterial ecology studied in continuous culture, Adv. Microbial Physiol., 11 (1974), pp. 165-212.

[JSTW] W. Jaeger, J. W. H. So, B. TANG, AND P. Waltman, Competition in the gradostat, J. Math. Biol., 25 (1987), pp. 23-42.

[K] J. KeEner, Principles of Applied Mathematics, Addison-Wesley, Reading, MA, 1987.

[L] A. W. Leung, Systems of Nonlinear Partial Differential Equations, Applications to Biology and Engineering, Kluwer Academic Publishers, Dordrecht, the Netherlands, 1989.

[LW] R. W. LOVITT AND J. W. T. WIMPENNY, The gradostat: $A$ bidirectional compound chemostat and Its applications in microbiological research, J. Gen. Microbial, 127 (1981), pp. 261-268.

[M1] H. MATANO, Existence of nontrivial unstable sets for equilibrium of strongly order-preserving systems, J. Fac. Sci. Univ. Tokyo Sect. IA Math., 30 (1984), pp. 605-673.

[M2] H. MATANO, Convergence of solutions of one dimensional semilinear parabolic equations, J. Math. Kyoto Univ., 18 (1978), pp. 221-227.

[P] P. POLACIK, Domain of attraction of equilibria and monotonocity properties of convergent trajectories in parabolic systems admitting a strong comparison principle, J. Reine Angew. Math., 400 (1989), pp. 32-56.

[PW] M. H. Protter and H. Weinberg, Maximum Principles in Differential Equations, Prentice-Hall, Englewood Cliffs, NJ, 1967.

[S] H. L. SMIth, Systems of ordinary differential equations which generate an order preserving flow, $A$ survey of results, SIAM Rev., 30 (1988), pp. 87-113. 
[Sm] J. SMOLleR, Shock Waves and Reaction Diffusion Equations, Springer-Verlag, New York, 1983.

[SoW] J. SO AND P. WALTMAN, A nonlinear boundary value problem arising from competition in the chemostat, Appl. Math. Comput., 32 (1989), pp. 169-183.

[STW] H. L. Smith, B. TANG, AND P. WaltMan, Competition in an n-vessel gradostat, SIAM J. Appl. Math., 5 (1991), pp. 1451-1471.

[SW] H. L. SMITH AND P. WALTMAN, The gradostat: A model of competition along a nutrient gradient, J. Microbial Ecology, 22 (1991), pp. 207-226.

[T] B. TANG, Mathematical investigations of growth of microorganisms in the gradostat, J. Math. Biol., 23 (1986), pp. 319-339.

[Th] H. R. THIEME, Convergence results and a Poincare Bendixson trichotomy for asymptotically autonomous differential equations, J. Math. Biol., 30 (1992), pp. 755-763.

[TW] P. A. TAYLOR AND J. L. WILliAM, Theoretical studies on the coexistence of competing species under continuous-flow conditions, Canad. J. Microbiology, 21 (1975), pp. 90-98.

[V] H. VELDCAMP, Ecological studies with the chemostat, Adv. Microbial Ecol., 1 (1977), pp. 59-95.

[W1] P. Waltman, Competition Models in Population Biology, Society for Industrial and Applied Mathematics, Philadelphia, 1983.

[W2] - Coexistence in chemostat like models, Rocky Mountain J. Math., 20 (1990), pp. 777-806.

[WHH] P. Waltman, S. P. HubBell, AND S. B. Hsu, Theoretical and experimental investigations of microbial competition in continuous culture, in Modeling and Differential Equations in Biology, T. Burton, ed., Marcel Dekker, New York, 1980. 
http://www.jstor.org

\title{
LINKED CITATIONS
}

\author{
- Page 1 of 2 -
}

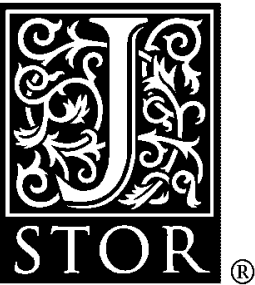

You have printed the following article:

On a System of Reaction-Diffusion Equations Arising From Competition in an Unstirred Chemostat

Sze-Bi Hsu; P. Waltman

SIAM Journal on Applied Mathematics, Vol. 53, No. 4. (Aug., 1993), pp. 1026-1044.

Stable URL:

http://links.jstor.org/sici?sici=0036-1399\%28199308\%2953\%3A4\%3C1026\%3AOASORE\%3E2.0.CO\%3B2-M

This article references the following linked citations. If you are trying to access articles from an off-campus location, you may be required to first logon via your library web site to access JSTOR. Please visit your library's website or contact a librarian to learn about options for remote access to JSTOR.

\section{References}

\author{
${ }^{\mathrm{FS}}$ Microbial Competition \\ A. G. Fredrickson; Gregory Stephanopoulos \\ Science, New Series, Vol. 213, No. 4511. (Aug. 28, 1981), pp. 972-979. \\ Stable URL: \\ http://links.jstor.org/sici?sici=0036-8075\%2819810828\%293\%3A213\%3A4511\%3C972\%3AMC\%3E2.0.CO\%3B2-S
}

\section{${ }^{\mathrm{HH}}$ Single-Nutrient Microbial Competition: Qualitative Agreement between Experimental and Theoretically Forecast Outcomes}

Stephen R. Hansen; Stephen P. Hubbell

Science, New Series, Vol. 207, No. 4438. (Mar. 28, 1980), pp. 1491-1493.

Stable URL:

http://links.jstor.org/sici?sici=0036-8075\%2819800328\%293\%3A207\%3A4438\%3C1491\%3ASMCQAB\%3E2.0.CO\%3B2-O

\section{${ }^{H H W}$ A Mathematical Theory for Single-Nutrient Competition in Continuous Cultures of Micro-Organisms}

S. B. Hsu; S. Hubbell; P. Waltman

SIAM Journal on Applied Mathematics, Vol. 32, No. 2. (Mar., 1977), pp. 366-383.

Stable URL:

http://links.jstor.org/sici?sici=0036-1399\%28197703\%2932\%3A2\%3C366\%3AAMTFSC\%3E2.0.CO\%3B2-H

NOTE: The reference numbering from the original has been maintained in this citation list. 
http://www.jstor.org

\section{LINKED CITATIONS \\ - Page 2 of 2 -}

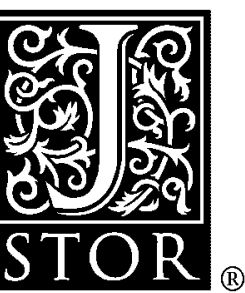

\section{${ }^{\mathrm{Hsu}}$ Limiting Behavior for Competing Species}

S. B. Hsu

SIAM Journal on Applied Mathematics, Vol. 34, No. 4. (Jun., 1978), pp. 760-763.

Stable URL:

http://links.jstor.org/sici?sici=0036-1399\%28197806\%2934\%3A4\%3C760\%3ALBFCS\%3E2.0.CO\%3B2-I

${ }^{\mathrm{S}}$ Systems of Ordinary Differential Equations Which Generate an Order Preserving Flow. A Survey of Results

Hal L. Smith

SIAM Review, Vol. 30, No. 1. (Mar., 1988), pp. 87-113.

Stable URL:

http://links.jstor.org/sici?sici=0036-1445\%28198803\%2930\%3A1\%3C87\%3ASOODEW\%3E2.0.CO\%3B2-U

\section{${ }^{\text {STW }}$ Competition in an n-Vessel Gradostat}

H. L. Smith; Betty Tang; Paul Waltman

SIAM Journal on Applied Mathematics, Vol. 51, No. 5. (Oct., 1991), pp. 1451-1471.

Stable URL:

http://links.jstor.org/sici?sici=0036-1399\%28199110\%2951\%3A5\%3C1451\%3ACIAG\%3E2.0.CO\%3B2-C

NOTE: The reference numbering from the original has been maintained in this citation list. 